\title{
Correction to: Statistical parameter optimization and modeling of photodegradation of methyl orange using a composite photocatalyst prepared by thermal synthesis
}

\author{
Samira Ghafoori ${ }^{1,2} \cdot$ Mohsen Nasirian $^{1} \cdot$ Rasha Al-Jamal $^{3} \cdot$ Fahad Abu Mallouh $^{2} \cdot$ Mehrab Mehrvar $^{1}$ (D) \\ Published online: 30 September 2020 \\ (C) Springer-Verlag GmbH Germany, part of Springer Nature 2020
}

Correction to: Environmental Science and Pollution Research

https://doi.org/10.1007/s11356-020-10301-5

Table 3 Proposed reaction scheme of the photocatalytic oxidation mechanism

\begin{tabular}{|c|c|c|c|}
\hline Step & Reaction & Reaction rate & Reference \\
\hline Activation & $\mathrm{TiO}_{2} \rightarrow^{\mathrm{hv}} \mathrm{TiO}_{2}+e^{-}+h^{+}$ & $r_{u v}=\varphi e_{\lambda}^{a}$ & $\begin{array}{l}\text { Satuf et al. } \\
\quad 2008\end{array}$ \\
\hline Recombination & $e^{-}+h^{+} \stackrel{k_{2}}{\rightarrow}$ heat & $k_{2}\left[e^{-}\right]\left[h^{+}\right]$ & $\begin{array}{l}\text { Satuf et al. } \\
2008\end{array}$ \\
\hline $\begin{array}{l}\text { Electron } \\
\text { trapping }\end{array}$ & $e^{-}+O_{2} \stackrel{k_{3}}{\rightarrow} O_{2}^{-}$ & $k_{3}\left[e^{-}\right]\left[O_{2}\right]$ & $\begin{array}{l}\text { Satuf et al. } \\
\quad 2008\end{array}$ \\
\hline Hole trapping & $h^{+}+\mathrm{H}_{2} \mathrm{O} \stackrel{k_{4}}{\rightarrow} \mathrm{OH}+\mathrm{H}^{+}$ & $k_{4}\left[h^{+}\right]\left[H_{2} O\right]$ & $\begin{array}{l}\text { Satuf et al. } \\
\quad 2008\end{array}$ \\
\hline Hydroxyl attack & $\mathrm{TOC}+{ }^{\bullet} \mathrm{OH} \stackrel{k_{5}}{\rightarrow} \ldots \rightarrow \mathrm{H}_{2} \mathrm{O}+\mathrm{CO}_{2}$ & $k_{5}[\mathrm{TOC}]\left[{ }^{\bullet} \mathrm{OH}\right]$ & Present study \\
\hline
\end{tabular}

Publisher's note Springer Nature remains neutral with regard to jurisdictional claims in published maps and institutional affiliations.

The online version of the original article can be found at https://doi.org/ $10.1007 / \mathrm{s} 11356-020-10301-5$

Mehrab Mehrvar

mmehrvar@ryerson.ca

1 Department of Chemical Engineering, Ryerson University, 350

Victoria Street, Toronto, ON M5B 2K3, Canada

2 Department of Petroleum Engineering, Australian College of Kuwait, P.O. Box 1411, 13015 Safat, Kuwait

3 Department of Mathematics, Australian College of Kuwait, P.O. Box 1411, 13015 Safat, Kuwait 